\title{
Oscillation results for difference equations with oscillating coefficients
}

\author{
George E Chatzarakis ${ }^{1}$, Hajnalka Péics ${ }^{2 *}$, Sandra Pinelas ${ }^{3}$ and loannis P Stavroulakis ${ }^{4}$
}

${ }^{\text {"Correspondence: }}$ peics@gf.uns.ac.rs

${ }^{2}$ Faculty of Civil Engineering, University of Novi Sad, Kozaračka 2/A, Subotica, 24000, Serbia Full list of author information is available at the end of the article

\section{Abstract}

Sufficient conditions which guarantee the oscillation of all solutions of difference equations with oscillating coefficients and several deviating arguments are presented. The corresponding difference equations of both retarded and advanced type are studied. Examples illustrating the results are also given.

\section{Introduction}

In the present paper, we study the oscillatory behavior of the solutions of the difference equation

$$
\Delta x(n)+\sum_{i=1}^{m} p_{i}(n) x\left(\tau_{i}(n)\right)=0, \quad n \in \mathbb{N}_{0},
$$

and the (dual) advanced difference equation

$$
\nabla x(n)-\sum_{i=1}^{m} p_{i}(n) x\left(\sigma_{i}(n)\right)=0, \quad n \in \mathbb{N},
$$

where $m \in \mathbb{N},\left\{p_{i}(n)\right\}_{n \in \mathbb{N}_{0}}, 1 \leq i \leq m$, are real sequences with oscillating terms, $\left\{\tau_{i}(n)\right\}_{n \in \mathbb{N}_{0}}$, $1 \leq i \leq m$, are sequences of integers such that

$$
\tau_{i}(n) \leq n-1, \quad n \in \mathbb{N}_{0}, \quad \text { and } \quad \lim _{n \rightarrow \infty} \tau_{i}(n)=\infty, \quad 1 \leq i \leq m,
$$

and $\left\{\sigma_{i}(n)\right\}_{n \in \mathbb{N}}, 1 \leq i \leq m$, are sequences of integers such that

$$
\sigma_{i}(n) \geq n+1, \quad n \in \mathbb{N}, 1 \leq i \leq m .
$$

Here, as usual, $\Delta$ denotes the forward difference operator $\Delta x(n)=x(n+1)-x(n)$ and $\nabla$ denotes the backward difference operator $\nabla x(n)=x(n)-x(n-1)$.

By a solution of $\left(\mathrm{E}_{\mathrm{R}}\right)$, we mean a sequence of real numbers $\{x(n)\}_{n \geq-w}$ which satisfies $\left(\mathrm{E}_{\mathrm{R}}\right)$ for all $n \in \mathbb{N}_{0}$. Here,

$$
w=-\min _{n \geq 0}\left\{\tau_{i}(n): 1 \leq i \leq m\right\} .
$$

() 2015 Chatzarakis et al.; licensee Springer. This is an Open Access article distributed under the terms of the Creative Commons Attribution License (http://creativecommons.org/licenses/by/4.0), which permits unrestricted use, distribution, and reproduction in any medium, provided the original work is properly credited. 
It is clear that, for each choice of real numbers $c_{-w}, c_{-w+1}, \ldots, c_{-1}, c_{0}$, there exists a unique solution $\{x(n)\}_{n \geq-w}$ of $\left(\mathrm{E}_{\mathrm{R}}\right)$ which satisfies the initial conditions $x(-w)=c_{-w}, x(-w+1)=$ $c_{-w+1}, \ldots, x(-1)=c_{-1}, x(0)=c_{0}$.

By a solution of $\left(\mathrm{E}_{\mathrm{A}}\right)$, we mean a sequence of real numbers $\{x(n)\}_{n \in \mathbb{N}_{0}}$ which satisfies $\left(\mathrm{E}_{\mathrm{A}}\right)$ for all $n \in \mathbb{N}$.

A solution $\{x(n)\}_{n \geq-w}\left(\{x(n)\}_{n \in \mathbb{N}_{0}}\right)$ of $\left(\mathrm{E}_{\mathrm{R}}\right)\left(\left(\mathrm{E}_{\mathrm{A}}\right)\right)$ is called oscillatory, if the terms $x(n)$ of the sequence are neither eventually positive nor eventually negative. Otherwise, the solution is said to be non-oscillatory.

In the last few decades, the oscillatory behavior of all solutions of difference equations has been extensively studied when the coefficients $p_{i}(n)$ are nonnegative. However, for the general case when $p_{i}(n)$ are allowed to oscillate, it is difficult to study the oscillation of $\left(\mathrm{E}_{\mathrm{R}}\right)$ $\left(\left(\mathrm{E}_{\mathrm{A}}\right)\right)$, since the difference $\Delta x(n)(\nabla x(n))$ of any non-oscillatory solution of $\left(\mathrm{E}_{\mathrm{R}}\right)\left(\left(\mathrm{E}_{\mathrm{A}}\right)\right)$ is always oscillatory. Therefore, the results on oscillation of difference and differential equations with oscillating coefficients are relatively scarce. Thus, a small number of paper are dealing with this case. See, for example, [1-17] and the references cited therein.

For $\left(\mathrm{E}_{\mathrm{R}}\right)$ and $\left(\mathrm{E}_{\mathrm{A}}\right)$ with oscillating coefficients, Bohner et al. [2, 3] established the following theorems.

Theorem 1.1 ([2, Theorem 2.4]) Assume that (1.1) holds and that the sequences $\left\{\tau_{i}(n)\right\}_{n \in \mathbb{N}_{0}}$ are increasing for all $i \in\{1, \ldots, m\}$. Suppose also that for each $i \in\{1, \ldots, m\}$ there exists a sequence $\left\{n_{i}(j)\right\}_{j \in \mathbb{N}}$ such that $\lim _{j \rightarrow \infty} n_{i}(j)=\infty$ and

$$
p_{k}(n) \geq 0, \quad n \in A=\bigcap_{i=1}^{m}\left\{\bigcup_{j \in \mathbb{N}}\left[\tau\left(\tau\left(n_{i}(j)\right)\right), n_{i}(j)\right] \cap \mathbb{N}\right\} \neq \emptyset, \quad 1 \leq k \leq m,
$$

where

$$
\tau(n)=\max _{1 \leq i \leq m} \tau_{i}(n), \quad n \in \mathbb{N}_{0}
$$

If, moreover,

$$
\limsup _{j \rightarrow \infty} \sum_{i=1}^{m} \sum_{q=\tau(n(j))}^{n(j)} p_{i}(q)>1
$$

where $n(j)=\min \left\{n_{i}(j): 1 \leq i \leq m\right\}$, then all solutions of $\left(\mathrm{E}_{\mathrm{R}}\right)$ oscillate.

Theorem 1.2 ([2, Theorem 3.4]) Assume that (1.2) holds and that the sequences $\left\{\sigma_{i}(n)\right\}_{n \in \mathbb{N}}$ are increasing for all $i \in\{1, \ldots, m\}$. Suppose also that for each $i \in\{1, \ldots, m\}$ there exists a sequence $\left\{n_{i}(j)\right\}_{j \in \mathbb{N}}$ such that $\lim _{j \rightarrow \infty} n_{i}(j)=\infty$ and

$$
p_{k}(n) \geq 0, \quad n \in B=\bigcap_{i=1}^{m}\left\{\bigcup_{j \in \mathbb{N}}\left[n_{i}(j), \sigma\left(\sigma\left(n_{i}(j)\right)\right)\right] \cap \mathbb{N}\right\} \neq \emptyset, \quad 1 \leq k \leq m
$$

where

$$
\sigma(n)=\min _{1 \leq i \leq m} \sigma_{i}(n), \quad n \in \mathbb{N}
$$


If, moreover,

$$
\limsup _{j \rightarrow \infty} \sum_{i=1}^{m} \sum_{q=n(j)}^{\sigma(n(j))} p_{i}(q)>1
$$

where $n(j)=\max \left\{n_{i}(j): 1 \leq i \leq m\right\}$, then all solutions of $\left(\mathrm{E}_{\mathrm{A}}\right)$ oscillate.

Theorem 1.3 ([3, Theorem 2.1]) Assume that (1.1) holds and that the sequences $\left\{\tau_{i}(n)\right\}_{n \in \mathbb{N}_{0}}$ are increasing for all $i \in\{1, \ldots, m\}$. Suppose also that for each $i \in\{1, \ldots, m\}$ there exists a sequence $\left\{n_{i}(j)\right\}_{j \in \mathbb{N}}$ such that $\lim _{j \rightarrow \infty} n_{i}(j)=\infty$,

$$
p_{k}(n) \geq 0, \quad n \in C=\bigcap_{i=1}^{m}\left\{\bigcup_{j \in \mathbb{N}}\left[\tau_{i}\left(\tau_{i}\left(n_{i}(j)\right)\right), n_{i}(j)\right] \cap \mathbb{N}\right\} \neq \emptyset, \quad 1 \leq k \leq m,
$$

and

$$
\limsup _{n \rightarrow \infty} \sum_{i=1}^{m} p_{i}(n)>0, \quad \text { for all } n \in C .
$$

If, moreover,

$$
\liminf _{j \rightarrow \infty} \sum_{i=1}^{m} \sum_{q=\tau_{i}\left(n_{i}(j)\right)}^{n_{i}(j)-1} p_{i}(q)>\frac{1}{e}
$$

then all solutions of $\left(\mathrm{E}_{\mathrm{R}}\right)$ oscillate.

Theorem 1.4 ([3, Theorem 3.1]) Assume that (1.2) holds and that the sequences $\left\{\sigma_{i}(n)\right\}_{n \in \mathbb{N}}$ are increasing for all $i \in\{1, \ldots, m\}$. Suppose also that for each $i \in\{1, \ldots, m\}$ there exists a sequence $\left\{n_{i}(j)\right\}_{j \in \mathbb{N}}$ such that $\lim _{j \rightarrow \infty} n_{i}(j)=\infty$,

$$
p_{k}(n) \geq 0, \quad n \in D=\bigcap_{i=1}^{m}\left\{\bigcup_{j \in \mathbb{N}}\left[n_{i}(j), \sigma_{i}\left(\sigma_{i}\left(n_{i}(j)\right)\right)\right] \cap \mathbb{N}\right\} \neq \emptyset, \quad 1 \leq k \leq m,
$$

and

$$
\limsup _{n \rightarrow \infty} \sum_{i=1}^{m} p_{i}(n)>0 \quad \text { for all } n \in D .
$$

If, moreover,

$$
\liminf _{j \rightarrow \infty} \sum_{i=1}^{m} \sum_{q=n_{i}(j)+1}^{\sigma_{i}\left(n_{i}(j)\right)} p_{i}(q)>\frac{1}{e}
$$

then all solutions of $\left(\mathrm{E}_{\mathrm{A}}\right)$ oscillate.

Recently, Berezansky et al. [1] and Chatzarakis et al. [4] established the following theorems.

Theorem 1.5 ([1, Theorem 8] and [4, Theorem 2.1]) Assume that (1.1) holds and the sequences $\left\{\tau_{i}(n)\right\}_{n \in \mathbb{N}_{0}}$ are increasing for all $i \in\{1, \ldots, m\}$ and the sequence $\tau$ is defined by 
(1.4). Suppose also that for each $i \in\{1, \ldots, m\}$ there exists a sequence $\left\{n_{i}(j)\right\}_{j \in \mathbb{N}}$ such that $\lim _{j \rightarrow \infty} n_{i}(j)=\infty$,

$$
p_{k}(n) \geq 0, \quad n \in A=\bigcap_{i=1}^{m}\left\{\bigcup_{j \in \mathbb{N}}\left[\tau\left(\tau\left(n_{i}(j)\right)\right), n_{i}(j)\right] \cap \mathbb{N}\right\} \neq \emptyset, \quad 1 \leq k \leq m .
$$

Set

$$
\alpha:=\liminf _{j \rightarrow \infty} \sum_{i=1}^{m} \sum_{q=\tau(n(j))}^{n(j)-1} p_{i}(q)
$$

where $n(j)=\min \left\{n_{i}(j): 1 \leq i \leq m\right\}$.

$$
\text { If } 0<\alpha \leq 1 / 2 \text {, and }
$$

$$
\limsup _{j \rightarrow \infty} \sum_{i=1}^{m} \sum_{q=\tau(n(j))}^{n(j)} p_{i}(q)>1-\frac{\alpha^{2}}{4(1-\alpha)},
$$

or

$$
\limsup _{j \rightarrow \infty} \sum_{i=1}^{m} \sum_{q=\tau(n(j))}^{n(j)} p_{i}(q)>1-\frac{1}{2}(1-\alpha-\sqrt{1-2 \alpha}),
$$

then all solutions of $\left(\mathrm{E}_{\mathrm{R}}\right)$ oscillate.

Theorem 1.6 ([1, Theorem 9] and [4, Theorem 3.1]) Assume (1.2) holds and the sequences $\left\{\sigma_{i}(n)\right\}_{n \in \mathbb{N}}$ are increasing for all $i \in\{1, \ldots, m\}$ and the sequence $\sigma$ is defined by (1.7). Suppose also that for each $i \in\{1, \ldots, m\}$ there exists a sequence $\left\{n_{i}(j)\right\}_{j \in \mathbb{N}}$ such that $\lim _{j \rightarrow \infty} n_{i}(j)=\infty$,

$$
p_{k}(n) \geq 0, \quad n \in B=\bigcap_{i=1}^{m}\left\{\bigcup_{j \in \mathbb{N}}\left[n_{i}(j), \sigma\left(\sigma\left(n_{i}(j)\right)\right)\right] \cap \mathbb{N}\right\} \neq \emptyset, \quad 1 \leq k \leq m .
$$

Set

$$
\alpha:=\liminf _{j \rightarrow \infty} \sum_{i=1}^{m} \sum_{q=n(j)+1}^{\sigma(n(j))} p_{i}(q)
$$

where $n(j)=\max \left\{n_{i}(j): 1 \leq i \leq m\right\}$.

$$
\text { If } 0<\alpha \leq 1 / 2 \text {, and }
$$

$$
\limsup _{j \rightarrow \infty} \sum_{i=1}^{m} \sum_{q=n(j)}^{\sigma(n(j))} p_{i}(q)>1-\frac{\alpha^{2}}{4(1-\alpha)},
$$

or

$$
\limsup _{j \rightarrow \infty} \sum_{i=1}^{m} \sum_{q=n(j)}^{\sigma(n(j))} p_{i}(q)>1-\frac{1}{2}(1-\alpha-\sqrt{1-2 \alpha}),
$$

then all solutions of $\left(\mathrm{E}_{\mathrm{A}}\right)$ oscillate. 
The authors study further $\left(\mathrm{E}_{\mathrm{R}}\right)$ and $\left(\mathrm{E}_{\mathrm{A}}\right)$ and derive new sufficient oscillation conditions. These conditions are the analogues of the oscillation conditions for the corresponding difference equations with positive coefficients, which were studied by Chatzarakis et al. [5]. Examples illustrate cases when the results of the paper imply oscillation while previously known results fail.

\section{Oscillation criteria}

\subsection{Retarded difference equations}

We present new sufficient conditions for the oscillation of all solutions of $\left(E_{R}\right)$, under the assumption that the sequences $\tau_{i}$ are increasing for all $i \in\{1, \ldots, m\}$.

Theorem 2.1 Assume that (1.1) holds, the sequences $\left\{\tau_{i}(n)\right\}_{n \in \mathbb{N}_{0}}$ are increasing for all $i \in$ $\{1, \ldots, m\}$ and the sequence $\tau$ is defined by (1.4). Suppose also that for each $i \in\{1, \ldots, m\}$ there exists a sequence $\left\{n_{i}(j)\right\}_{j \in \mathbb{N}}$ such that $\lim _{j \rightarrow \infty} n_{i}(j)=\infty$,

$$
p_{k}(n)>0, \quad n \in A=\bigcap_{i=1}^{m}\left\{\bigcup_{j \in \mathbb{N}}\left[\tau\left(\tau\left(n_{i}(j)\right)\right), n_{i}(j)\right] \cap \mathbb{N}\right\} \neq \emptyset, \quad 1 \leq k \leq m,
$$

with

$$
\liminf _{n \rightarrow \infty}\left\{p_{k}(n): n \in A\right\}>0, \quad 1 \leq k \leq m .
$$

If, moreover,

$$
\left[\prod_{i=1}^{m}\left(\sum_{\ell=1}^{m} \liminf _{j \rightarrow \infty} \sum_{k=\tau_{\ell}(n(j))}^{n(j)-1} p_{i}(k)\right)\right]^{1 / m}>\frac{1}{e}
$$

where $n(j)=\min \left\{n_{i}(j): 1 \leq i \leq m\right\}$, then all solutions of $\left(\mathrm{E}_{\mathrm{R}}\right)$ oscillate.

Proof Assume, for the sake of contradiction, that $\{x(n)\}_{n \geq-w}$ is an eventually positive solution of $\left(\mathrm{E}_{\mathrm{R}}\right)$. Then there exists $j_{0} \in \mathbb{N}$ such that

$$
\begin{aligned}
& p_{k}(n)>0 \quad \text { for all } n \in \bigcap_{i=1}^{m}\left[\tau\left(\tau\left(n_{i}\left(j_{0}\right)\right)\right), n_{i}\left(j_{0}\right)\right] \cap \mathbb{N}, \quad 1 \leq k \leq m, \\
& x\left(\tau_{k}(n)\right)>0 \quad \text { for all } n \in \bigcap_{i=1}^{m}\left[\tau\left(\tau\left(n_{i}\left(j_{0}\right)\right)\right), n_{i}\left(j_{0}\right)\right] \cap \mathbb{N}, \quad 1 \leq k \leq m .
\end{aligned}
$$

Therefore, by $\left(E_{R}\right)$ we have

$$
x(n+1)-x(n)=-\sum_{i=1}^{m} p_{i}(n) x\left(\tau_{i}(n)\right)<0,
$$

for every $n \in \bigcap_{i=1}^{m}\left[\tau\left(\tau\left(n_{i}\left(j_{0}\right)\right)\right), n_{i}\left(j_{0}\right)\right] \cap \mathbb{N}$. This guarantees that the sequence $x$ is strictly decreasing on $\bigcap_{i=1}^{m}\left[\tau\left(\tau\left(n_{i}\left(j_{0}\right)\right)\right), n_{i}\left(j_{0}\right)\right] \cap \mathbb{N}$.

Set

$$
n\left(j_{0}\right)=\min \left\{n_{i}\left(j_{0}\right): 1 \leq i \leq m\right\}
$$


and

$$
z_{i}\left(n\left(j_{0}\right)\right)=\frac{x\left(\tau_{i}\left(n\left(j_{0}\right)\right)\right)}{x\left(n\left(j_{0}\right)\right)}, \quad 1 \leq i \leq m
$$

with

$$
\varphi_{i}=\liminf _{j_{0} \rightarrow \infty} z_{i}\left(n\left(j_{0}\right)\right), \quad 1 \leq i \leq m .
$$

It is obvious that

$$
z_{i}\left(n\left(j_{0}\right)\right)>1 \quad \text { and } \quad \varphi_{i} \geq 1 \quad \text { for } i=1,2, \ldots, m
$$

Dividing both sides of $\left(\mathrm{E}_{\mathrm{R}}\right)$ by $x\left(n\left(j_{0}\right)\right)$, we obtain

$$
\frac{\Delta x\left(n\left(j_{0}\right)\right)}{x\left(n\left(j_{0}\right)\right)}+\sum_{i=1}^{m} p_{i}\left(n\left(j_{0}\right)\right) \frac{x\left(\tau_{i}\left(n\left(j_{0}\right)\right)\right)}{x\left(n\left(j_{0}\right)\right)}=0
$$

or

$$
\frac{\Delta x\left(n\left(j_{0}\right)\right)}{x\left(n\left(j_{0}\right)\right)}+\sum_{i=1}^{m} p_{i}\left(n\left(j_{0}\right)\right) z_{i}\left(n\left(j_{0}\right)\right)=0 .
$$

Summing up (2.5) from $\tau_{\rho}\left(n\left(j_{0}\right)\right)$ to $n\left(j_{0}\right)-1$ for $\rho=1,2, \ldots, m$, we find

$$
\sum_{j=\tau_{\rho}\left(n\left(j_{0}\right)\right)}^{n\left(j_{0}\right)-1} \frac{\Delta x(j)}{x(j)}+\sum_{i=1}^{m} \sum_{j=\tau_{\rho}\left(n\left(j_{0}\right)\right)}^{n\left(j_{0}\right)-1} p_{i}(j) z_{i}(j)=0
$$

But

$$
\begin{aligned}
\sum_{j=\tau_{\rho}\left(n\left(j_{0}\right)\right)}^{n\left(j_{0}\right)-1} \frac{\Delta x(j)}{x(j)} & =\sum_{j=\tau_{\rho}\left(n\left(j_{0}\right)\right)}^{n\left(j_{0}\right)-1}\left(\frac{x(j+1)}{x(j)}-1\right) \\
& \geq \sum_{j=\tau_{\rho}\left(n\left(j_{0}\right)\right)}^{n\left(j_{0}\right)-1}\left(1+\ln \frac{x(j+1)}{x(j)}-1\right)=\ln \frac{x\left(n\left(j_{0}\right)\right)}{x\left(\tau_{\rho}\left(n\left(j_{0}\right)\right)\right)}
\end{aligned}
$$

or

$$
\sum_{j=\tau_{\rho}\left(n\left(j_{0}\right)\right)}^{n\left(j_{0}\right)-1} \frac{\Delta x(j)}{x(j)} \geq-\ln z_{\rho}\left(n\left(j_{0}\right)\right) .
$$

Combining (2.6) and (2.7), we obtain

$$
-\ln z_{\rho}\left(n\left(j_{0}\right)\right)+\sum_{i=1}^{m} \sum_{j=\tau_{\rho}\left(n\left(j_{0}\right)\right)}^{n\left(j_{0}\right)-1} p_{i}(j) z_{i}(j) \leq 0
$$


or

$$
\ln z_{\rho}\left(n\left(j_{0}\right)\right) \geq \sum_{i=1}^{m} \sum_{j=\tau \rho}^{n\left(n\left(j_{0}\right)\right)} p_{i}(j) z_{i}(j), \quad \rho=1,2, \ldots, m
$$

Now we will show that $\varphi_{i}<\infty$ for $i=1,2, \ldots, m$. Indeed, assume that $\varphi_{i}=\infty$ for some $i$, $i=1,2, \ldots, m$. By using (2.5) we have

$$
\frac{x\left(n\left(j_{0}\right)+1\right)}{x\left(n\left(j_{0}\right)\right)}-1+\sum_{i=1}^{m} p_{i}\left(n\left(j_{0}\right)\right) z_{i}\left(n\left(j_{0}\right)\right)=0
$$

or

$$
\frac{x\left(n\left(j_{0}\right)+1\right)}{x\left(n\left(j_{0}\right)\right)}+\sum_{i=1}^{m} p_{i}\left(n\left(j_{0}\right)\right) z_{i}\left(n\left(j_{0}\right)\right)=1
$$

Consequently,

$$
\liminf _{j_{0} \rightarrow \infty}\left[\frac{x\left(n\left(j_{0}\right)+1\right)}{x\left(n\left(j_{0}\right)\right)}+\sum_{i=1}^{m} p_{i}\left(n\left(j_{0}\right)\right) z_{i}\left(n\left(j_{0}\right)\right)\right]=1
$$

and therefore

$$
\liminf _{j_{0} \rightarrow \infty} \frac{x\left(n\left(j_{0}\right)+1\right)}{x\left(n\left(j_{0}\right)\right)}+\sum_{i=1}^{m} \liminf _{j_{0} \rightarrow \infty}\left(p_{i}\left(n\left(j_{0}\right)\right) z_{i}\left(n\left(j_{0}\right)\right)\right) \leq 1
$$

or

$$
\liminf _{j_{0} \rightarrow \infty} \frac{x\left(n\left(j_{0}\right)+1\right)}{x\left(n\left(j_{0}\right)\right)}+\sum_{i=1}^{m} \liminf _{j_{0} \rightarrow \infty} p_{i}\left(n\left(j_{0}\right) \liminf _{j_{0} \rightarrow \infty} z_{i}\left(n\left(j_{0}\right)\right) \leq 1\right.
$$

In view of (2.1) and taking into account the fact that $\varphi_{i}=\infty$ for some $i$, the above inequality is false. Hence $\varphi_{i}<\infty$ for $i=1,2, \ldots, m$.

Taking the limit inferiors on both sides of the above inequalities (2.8), we obtain

$$
\ln \varphi_{\rho} \geq \sum_{i=1}^{m} \varphi_{i}\left(\liminf _{j_{0} \rightarrow \infty} \sum_{k=\tau_{\rho}\left(n\left(j_{0}\right)\right)}^{n\left(j_{0}\right)-1} p_{i}(k)\right), \quad \rho=1,2, \ldots, m,
$$

and by adding we find

$$
\sum_{i=1}^{m} \ln \varphi_{i} \geq \sum_{i=1}^{m} \varphi_{i}\left(\sum_{j=1}^{m} \liminf _{j_{0} \rightarrow \infty} \sum_{k=\tau_{j}\left(n\left(j_{0}\right)\right)}^{n\left(j_{0}\right)-1} p_{i}(k)\right)
$$

Set

$$
f\left(\varphi_{1}, \varphi_{2}, \ldots, \varphi_{m}\right) \equiv \sum_{i=1}^{m} \ln \varphi_{i}-\sum_{i=1}^{m} \varphi_{i}\left(\sum_{j=1}^{m} \liminf _{j_{0} \rightarrow \infty} \sum_{k=\tau_{j}\left(n\left(j_{0}\right)\right)}^{n\left(j_{0}\right)-1} p_{i}(k)\right) .
$$


Clearly

$$
f\left(\varphi_{1}, \varphi_{2}, \ldots, \varphi_{m}\right) \geq 0 \quad \text { for all } \varphi_{1}, \varphi_{2}, \ldots, \varphi_{m} \geq 1
$$

Since

$$
\frac{\partial f}{\partial \varphi_{i}}=\frac{1}{\varphi_{i}}-\sum_{j=1}^{m} \liminf _{j_{0} \rightarrow \infty} \sum_{k=\tau_{j}\left(n\left(j_{0}\right)\right)}^{n\left(j_{0}\right)-1} p_{i}(k)=0
$$

for

$$
\varphi_{i}=\frac{1}{\sum_{j=1}^{m} \liminf _{j_{0} \rightarrow \infty} \sum_{k=\tau_{j}\left(n\left(j_{0}\right)\right)}^{n\left(j_{0}\right)-1} p_{i}(k)}, \quad i=1,2, \ldots, m,
$$

the function $f$ has a maximum at the critical point

$$
\left(\frac{1}{\sum_{j=1}^{m} \liminf _{j_{0} \rightarrow \infty} \sum_{k=\tau_{j}\left(n\left(j_{0}\right)\right)}^{n\left(j_{0}\right)-1} p_{1}(k)}, \ldots, \frac{1}{\sum_{j=1}^{m} \liminf _{j_{0} \rightarrow \infty} \sum_{k=\tau_{j}\left(n\left(j_{0}\right)\right)}^{n\left(j_{j}\right)-1} p_{m}(k)}\right)
$$

because the quadratic form

$$
\sum_{i, j=1}^{m} \frac{\partial^{2} f}{\partial \varphi_{i} \partial \varphi_{j}} \alpha_{i} \alpha_{j}=-\sum_{i=1}^{m} \frac{\alpha_{i}^{2}}{\varphi_{i}^{2}}<0
$$

Since $f\left(\varphi_{1}, \varphi_{2}, \ldots, \varphi_{m}\right) \geq 0$, the maximum of $f$ at the critical point should be nonnegative. That is,

$$
\sum_{i=1}^{m}\left[-\ln \left(\sum_{j=1}^{m} \liminf _{j_{0} \rightarrow \infty} \sum_{k=\tau_{j}\left(n\left(j_{0}\right)\right)}^{n\left(j_{0}\right)-1} p_{i}(k)\right)\right]-m \geq 0
$$

i.e.,

$$
\max _{\varphi_{i} \geq 1} f\left(\varphi_{1}, \varphi_{2}, \ldots, \varphi_{m}\right)=-\ln \prod_{i=1}^{m}\left(\sum_{j=1}^{m} \liminf _{j_{0} \rightarrow \infty} \sum_{k=\tau_{j}\left(n\left(j_{0}\right)\right)}^{n\left(j_{0}\right)-1} p_{i}(k)\right)-m .
$$

Hence

$$
\prod_{i=1}^{m}\left(\sum_{j=1}^{m} \liminf _{j_{0} \rightarrow \infty} \sum_{k=\tau_{j}\left(n\left(j_{0}\right)\right)}^{n\left(j_{0}\right)-1} p_{i}(k)\right) \leq \frac{1}{e^{m}}
$$

or

$$
\left[\prod_{i=1}^{m}\left(\sum_{j=1}^{m} \liminf _{j_{0} \rightarrow \infty} \sum_{k=\tau_{j}\left(n\left(j_{0}\right)\right)}^{n\left(j_{0}\right)-1} p_{i}(k)\right)\right]^{1 / m} \leq \frac{1}{e}
$$

which contradicts (2.2).

The proof of the theorem is complete. 
Theorem 2.2 Assume that (1.1) holds, the sequences $\left\{\tau_{i}(n)\right\}_{n \in \mathbb{N}_{0}}$ are increasing for all $i \in$ $\{1, \ldots, m\}$ and the sequence $\tau$ is defined by (1.4). Suppose also that for each $i \in\{1, \ldots, m\}$ there exists a sequence $\left\{n_{i}(j)\right\}_{j \in \mathbb{N}}$ such that $\lim _{j \rightarrow \infty} n_{i}(j)=\infty$,

$$
p_{k}(n)>0, \quad n \in A=\bigcap_{i=1}^{m}\left\{\bigcup_{j \in \mathbb{N}}\left[\tau\left(\tau\left(n_{i}(j)\right)\right), n_{i}(j)\right] \cap \mathbb{N}\right\} \neq \emptyset, \quad 1 \leq k \leq m,
$$

with

$$
\liminf _{n \rightarrow \infty}\left\{p_{k}(n): n \in A\right\}>0, \quad 1 \leq k \leq m .
$$

If, moreover,

$$
\begin{aligned}
& \frac{1}{m} \sum_{i=1}^{m} \liminf _{j \rightarrow \infty} \sum_{k=\tau_{i}(n(j))}^{n(j)-1} p_{i}(k) \\
& +\frac{2}{m} \sum_{\substack{i<\ell \\
i, \ell=1}}^{m}\left(\liminf _{j \rightarrow \infty} \sum_{k=\tau_{\ell}(n(j))}^{n(j)-1} p_{i}(k) \times \liminf _{j \rightarrow \infty} \sum_{k=\tau_{i}(n(j))}^{n(j)-1} p_{\ell}(k)\right)^{1 / 2}>\frac{1}{e},
\end{aligned}
$$

where $n(j)=\min \left\{n_{i}(j): 1 \leq i \leq m\right\}$, then all solutions of $\left(\mathrm{E}_{\mathrm{R}}\right)$ oscillate.

Proof Assume, for the sake of contradiction, that $\{x(n)\}_{n \geq-w}$ is an eventually positive solution of $\left(\mathrm{E}_{\mathrm{R}}\right)$. Then there exists $j_{0} \in \mathbb{N}$ such that

$$
\begin{aligned}
& p_{k}(n)>0 \quad \text { for all } n \in \bigcap_{i=1}^{m}\left[\tau\left(\tau\left(n_{i}\left(j_{0}\right)\right)\right), n_{i}\left(j_{0}\right)\right] \cap \mathbb{N}, \quad 1 \leq k \leq m, \\
& x\left(\tau_{k}(n)\right)>0 \quad \text { for all } n \in \bigcap_{i=1}^{m}\left[\tau\left(\tau\left(n_{i}\left(j_{0}\right)\right)\right), n_{i}\left(j_{0}\right)\right] \cap \mathbb{N}, \quad 1 \leq k \leq m .
\end{aligned}
$$

Therefore, by $\left(E_{R}\right)$ we have

$$
x(n+1)-x(n)=-\sum_{i=1}^{m} p_{i}(n) x\left(\tau_{i}(n)\right)<0,
$$

for every $n \in \bigcap_{i=1}^{m}\left[\tau\left(\tau\left(n_{i}\left(j_{0}\right)\right)\right), n_{i}\left(j_{0}\right)\right] \cap \mathbb{N}$. This guarantees that the sequence $x$ is strictly decreasing on $\bigcap_{i=1}^{m}\left[\tau\left(\tau\left(n_{i}\left(j_{0}\right)\right)\right), n_{i}\left(j_{0}\right)\right] \cap \mathbb{N}$.

Taking into account the fact that $\varphi_{i}<\infty$ for $i=1,2, \ldots, m$ (see Theorem 2.1), by using (2.9) and the fact that

$$
\frac{1}{e}>\frac{\ln \varphi_{\rho}}{\varphi_{\rho}}, \quad \rho=1,2, \ldots, m
$$

we obtain

$$
\frac{1}{e}>\sum_{i=1}^{m}\left(\liminf _{j_{0} \rightarrow \infty} \sum_{k=\tau_{\ell}\left(n\left(j_{0}\right)\right)}^{n\left(j_{0}\right)-1} p_{i}(k)\right) \frac{\varphi_{i}}{\varphi_{\ell}}, \quad \ell=1,2, \ldots, m .
$$


Adding these inequalities we have

$$
\begin{aligned}
\frac{m}{e} \geq & \sum_{i=1}^{m}\left(\liminf _{j_{0} \rightarrow \infty} \sum_{k=\tau_{i}\left(n\left(j_{0}\right)\right)}^{n\left(j_{0}\right)-1} p_{i}(k)\right) \\
& +\sum_{i<\ell}^{m}\left[\left(\liminf _{j_{0} \rightarrow \infty} \sum_{k=\tau_{\ell}\left(n\left(j_{0}\right)\right)}^{n\left(j_{0}\right)-1} p_{i}(k)\right) \frac{\varphi_{i}}{\varphi_{\ell}}+\left(\liminf _{j_{0} \rightarrow \infty} \sum_{k=\tau_{i}\left(n\left(j_{0}\right)\right)}^{n\left(j_{0}\right)-1} p_{\ell}(k)\right) \frac{\varphi_{\ell}}{\varphi_{i}}\right] \\
\geq & \sum_{i=1}^{m}\left(\liminf _{j_{0} \rightarrow \infty} \sum_{k=\tau_{i}\left(n\left(j_{0}\right)\right)}^{n\left(j_{0}\right)-1} p_{i}(k)\right) \\
& +2 \sum_{\substack{i<\ell \\
i, \ell=1}}^{m}\left[\left(\liminf _{j_{0} \rightarrow \infty} \sum_{k=\tau_{\ell}\left(n\left(j_{0}\right)\right)} p_{i}(k)\right) \times\left(\liminf _{j_{0} \rightarrow \infty} \sum_{k=\tau_{i}\left(n\left(j_{0}\right)\right)}^{n\left(j_{0}\right)-1} p_{\ell}(k)\right)\right]^{1 / 2}
\end{aligned}
$$

or

$$
\sum_{i=1}^{m} \liminf _{j_{0} \rightarrow \infty} \sum_{k=\tau_{i}\left(n\left(j_{0}\right)\right)}^{n\left(j_{0}\right)-1} p_{i}(k)+2 \sum_{\substack{i<l \\ i, l=1}}^{m} \sqrt{\liminf _{j_{0} \rightarrow \infty} \sum_{k=\tau_{\ell}\left(n\left(j_{0}\right)\right)}^{n\left(j_{0}\right)-1} p_{i}(k) \times \liminf _{j_{0} \rightarrow \infty} \sum_{k=\tau_{i}\left(n\left(j_{0}\right)\right)}^{n\left(j_{0}\right)-1} p_{\ell}(k)} \leq \frac{m}{e},
$$

which contradicts (2.10).

The proof of the theorem is complete.

A slight modification in the proofs of Theorem 2.1 or Theorem 2.2 leads to the following result about retarded difference inequalities.

Theorem 2.3 Assume that all conditions of Theorem 2.1 or Theorem 2.2 hold. Then

(i) the difference inequality

$$
\Delta x(n)+\sum_{i=1}^{m} p_{i}(n) x\left(\tau_{i}(n)\right) \leq 0, \quad n \in \mathbb{N}_{0}
$$

has no eventually positive solutions;

(ii) the difference inequality

$$
\Delta x(n)+\sum_{i=1}^{m} p_{i}(n) x\left(\tau_{i}(n)\right) \geq 0, \quad n \in \mathbb{N}_{0}
$$

has no eventually negative solutions.

\subsection{Advanced difference equations}

Similar oscillation theorems for the (dual) advanced difference equation $\left(\mathrm{E}_{\mathrm{A}}\right)$ can be derived easily. The proofs of these theorems are omitted, since they follow a similar procedure as in Section 2.1.

Theorem 2.4 Assume that (1.2) holds, the sequences $\left\{\sigma_{i}(n)\right\}_{n \in \mathbb{N}}$ are increasing for all $i \in$ $\{1, \ldots, m\}$ and the sequence $\sigma$ is defined by (1.7). Suppose also that for each $i \in\{1, \ldots, m\}$ 
there exists a sequence $\left\{n_{i}(j)\right\}_{j \in \mathbb{N}}$ such that $\lim _{j \rightarrow \infty} n_{i}(j)=\infty$,

$$
p_{k}(n) \geq 0, \quad n \in B=\bigcap_{i=1}^{m}\left\{\bigcup_{j \in \mathbb{N}}\left[n_{i}(j), \sigma\left(\sigma\left(n_{i}(j)\right)\right)\right] \cap \mathbb{N}\right\} \neq \emptyset, \quad 1 \leq k \leq m,
$$

with

$$
\liminf _{n \rightarrow \infty}\left\{p_{k}(n): n \in B\right\}>0, \quad 1 \leq k \leq m .
$$

If, moreover,

$$
\left[\prod_{i=1}^{m}\left(\sum_{\ell=1}^{m} \liminf _{j \rightarrow \infty} \sum_{k=n(j)+1}^{\sigma_{\ell}(n(j))} p_{i}(k)\right)\right]^{1 / m}>\frac{1}{e}
$$

where $n(j)=\max \left\{n_{i}(j): 1 \leq i \leq m\right\}$, then all solutions of $\left(\mathrm{E}_{\mathrm{A}}\right)$ oscillate.

Theorem 2.5 Assume that (1.2) holds, the sequences $\left\{\sigma_{i}(n)\right\}_{n \in \mathbb{N}}$ are increasing for all $i \in$ $\{1, \ldots, m\}$ and the sequence $\sigma$ is defined by (1.7). Suppose also that for each $i \in\{1, \ldots, m\}$ there exists a sequence $\left\{n_{i}(j)\right\}_{j \in \mathbb{N}}$ such that $\lim _{j \rightarrow \infty} n_{i}(j)=\infty$,

$$
p_{k}(n) \geq 0, \quad n \in B=\bigcap_{i=1}^{m}\left\{\bigcup_{j \in \mathbb{N}}\left[n_{i}(j), \sigma\left(\sigma\left(n_{i}(j)\right)\right)\right] \cap \mathbb{N}\right\} \neq \emptyset, \quad 1 \leq k \leq m,
$$

with

$$
\liminf _{n \rightarrow \infty}\left\{p_{k}(n): n \in B\right\}>0, \quad 1 \leq k \leq m .
$$

If, moreover,

$$
\begin{aligned}
& \frac{1}{m} \sum_{i=1}^{m} \liminf _{j \rightarrow \infty} \sum_{k=n(j)+1}^{\sigma_{i}(n(j))} p_{i}(k) \\
& \quad+\frac{2}{m} \sum_{\substack{i<\ell \\
i, \ell=1}}^{m}\left(\liminf _{j \rightarrow \infty} \sum_{k=n(j)+1}^{\sigma_{\ell}(n(j))} p_{i}(k) \times \liminf _{j \rightarrow \infty} \sum_{k=n(j)+1}^{\sigma_{i}(n(j))} p_{\ell}(k)\right)^{1 / 2}>\frac{1}{e},
\end{aligned}
$$

where $n(j)=\max \left\{n_{i}(j): 1 \leq i \leq m\right\}$, then all solutions of $\left(\mathrm{E}_{\mathrm{A}}\right)$ oscillate.

A slight modification in the proofs of Theorems 2.4 or Theorem 2.5 leads to the following result about advanced difference inequalities.

Theorem 2.6 Assume that all conditions of Theorem 2.4 or Theorem 2.5 hold. Then

(i) the difference inequality

$$
\nabla x(n)-\sum_{i=1}^{m} p_{i}(n) x\left(\sigma_{i}(n)\right) \geq 0, \quad n \in \mathbb{N},
$$

has no eventually positive solutions; 
(ii) the difference inequality

$$
\nabla x(n)-\sum_{i=1}^{m} p_{i}(n) x\left(\sigma_{i}(n)\right) \leq 0, \quad n \in \mathbb{N},
$$

has no eventually negative solutions.

\subsection{Special cases}

In the case where $p_{i}, i=1,2, \ldots, m$, are oscillating real constants and $\tau_{i}$ are constant retarded arguments of the form $\tau_{i}(n)=n-k_{i}$, ( $\sigma_{i}$ are constant advanced arguments of the form $\left.\sigma_{i}(n)=n+k_{i}\right), k_{i} \in \mathbb{N}, i=1,2, \ldots, m,\left(\mathrm{E}_{\mathrm{R}}\right)\left(\left(\mathrm{E}_{\mathrm{A}}\right)\right)$ takes the form

$$
\Delta x(n)+\sum_{i=1}^{m} p_{i} x\left(n-k_{i}\right)=0, \quad n \in \mathbb{N}_{0} \quad\left(\nabla x(n)-\sum_{i=1}^{m} p_{i} x\left(n+k_{i}\right)=0, \quad n \in \mathbb{N}\right) .
$$

For this equation, as a consequence of Theorem 2.1 (Theorem 2.4) and Theorem 2.2 (Theorem 2.5), we have the following corollary.

Corollary 2.1 Assume that

$$
\left[\prod_{i=1}^{m} p_{i}\right]^{1 / m}\left(\sum_{i=1}^{m} k_{i}\right)>\frac{1}{e}
$$

or

$$
\frac{1}{m}\left(\sum_{i=1}^{m} \sqrt{p_{i} k_{i}}\right)^{2}>\frac{1}{e}
$$

Then all solutions of $(\mathrm{E})$ oscillate.

\section{Examples}

The following two examples illustrate that the conditions for oscillations (2.14) and (2.15) are independent. They are chosen in such a way that only one of them is satisfied. Also, in Example 3.1, Theorem 1.1 [2], Theorem 1.3 [3] and Theorem 1.5 [1, 4] do not imply oscillation, and neither do Theorem 1.2 [2], Theorem 1.4 [3] and Theorem 1.6 [1, 4] in Example 3.2. According to conditions (2.14) and (2.15) oscillation is established in Example 3.1 and Example 3.2, respectively.

Example 3.1 Consider the retarded difference equation

$$
\Delta x(n)+p_{1}(n) x(n-1)+p_{2}(n) x(n-2)=0, \quad n \in \mathbb{N}_{0},
$$

where $p_{1}(n)$ and $p_{2}(n)$ are oscillating coefficients, as shown in Figure 1.

In view of (1.4), it is obvious that $\tau(n)=n-1$. Observe that for

$$
n_{1}(j)=12 j+5, \quad j \in \mathbb{N}
$$



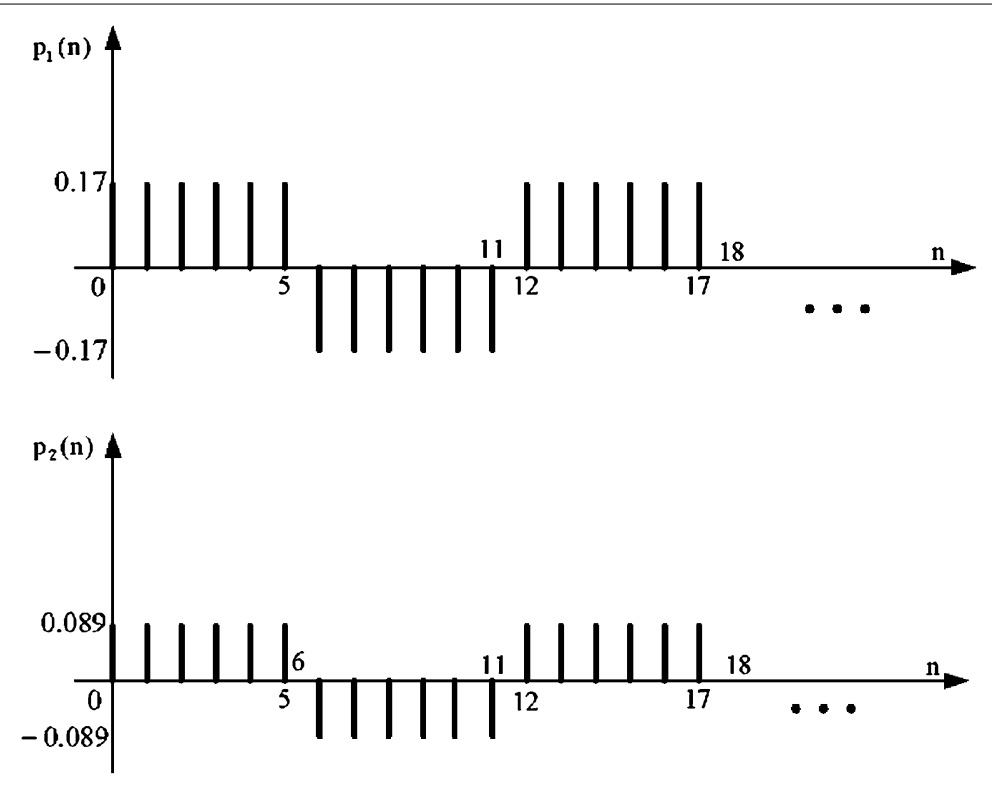

Figure 1 Coefficients of Example 3.1.

we have $p_{1}(n)>0$ for every $n \in A$, where

$$
A=\bigcup_{j \in \mathbb{N}}\left[\tau\left(\tau\left(n_{1}(j)\right)\right), n_{1}(j)\right] \cap \mathbb{N}=\bigcup_{j \in \mathbb{N}}[12 j+3,12 j+5] \cap \mathbb{N} .
$$

Also, for

$$
n_{2}(j)=12 j+4, \quad j \in \mathbb{N},
$$

we have $p_{2}(n)>0$ for every $n \in B$, where

$$
B=\bigcup_{j \in \mathbb{N}}\left[\tau\left(\tau\left(n_{2}(j)\right)\right), n_{2}(j)\right] \cap \mathbb{N}=\bigcup_{j \in \mathbb{N}}[12 j+2,12 j+4] \cap \mathbb{N} .
$$

Therefore

$$
p_{1}(n)>0 \quad \text { and } \quad p_{2}(n)>0 \quad \text { for all } n \in A \cap B=\bigcup_{j \in \mathbb{N}}[12 j+3,12 j+4] \cap \mathbb{N} \neq \emptyset,
$$

that is, (1.3) is satisfied. Moreover, the computation immediately implies that

$$
\left[\prod_{i=1}^{2} p_{i}\right]^{1 / 2}\left(\sum_{i=1}^{2} k_{i}\right)=\sqrt{\frac{17}{100} \cdot \frac{89}{1,000}} \cdot(1+2) \simeq 0.369012194>\frac{1}{e},
$$

that is, condition (2.14) of Corollary 2.1 is satisfied and therefore all solutions of (3.1) oscillate. Observe, however, that

$$
\frac{1}{2}\left(\sum_{i=1}^{2} \sqrt{p_{i} k_{i}}\right)^{2}=\frac{1}{2}\left(\sqrt{\frac{17}{100} \cdot 1}+\sqrt{\frac{89}{1,000} \cdot 2}\right)^{2} \simeq 0.347954016<\frac{1}{e},
$$

that is, condition (2.15) of Corollary 2.1 is not satisfied. 
Also,

$$
\begin{aligned}
& n(j)=\min \left\{n_{i}(j): 1 \leq i \leq 2\right\}=12 j+4, \quad j \in \mathbb{N}, \\
& \alpha=\liminf _{j \rightarrow \infty} \sum_{i=1}^{m} \sum_{q=\tau(n(j))}^{n(j)-1} p_{i}(q) \\
& =\liminf _{j \rightarrow \infty}\left[\sum_{q=12 j+3}^{12 j+3} p_{1}(q)+\sum_{q=12 j+3}^{12 j+3} p_{2}(q)\right]=\frac{17}{100}+\frac{89}{1,000}=0.259, \\
& \limsup _{j \rightarrow \infty} \sum_{i=1}^{2} \sum_{q=\tau(n(j))}^{n(j)} p_{i}(q) \\
& =\limsup _{j \rightarrow \infty}\left[\sum_{q=12 j+3}^{12 j+4} p_{1}(q)+\sum_{q=12 j+3}^{12 j+4} p_{2}(q)\right]=2 \cdot \frac{17}{100}+2 \cdot \frac{89}{1,000}=0.518 .
\end{aligned}
$$

Observe that

$$
\begin{aligned}
& 0.518<1 \\
& 0.518<1-\frac{\alpha^{2}}{4(1-\alpha)} \simeq 0.977368083 \\
& 0.518<1-\frac{1}{2}(1-\alpha-\sqrt{1-2 \alpha}) \simeq 0.976631099 .
\end{aligned}
$$

Therefore the conditions (1.5), (1.16), and (1.17) are not satisfied.

On the other hand, $p_{1}(n)>0$ for every $n \in A^{\prime}=A$ and $p_{2}(n)>0$ for every $n \in B^{\prime}$, where

$$
B^{\prime}=\bigcup_{j \in \mathbb{N}}\left[\tau_{2}\left(\tau_{2}\left(n_{2}(j)\right)\right), n_{2}(j)\right] \cap \mathbb{N}=\bigcup_{j \in \mathbb{N}}[12 j, 12 j+4] \cap \mathbb{N} .
$$

Hence

$$
p_{1}(n)>0 \quad \text { and } \quad p_{2}(n)>0 \quad \text { for all } n \in A^{\prime} \cap B^{\prime}=A \cap B \neq \emptyset,
$$

that is, condition (1.9) is satisfied. In view of this, we have

$$
\begin{aligned}
\liminf _{j \rightarrow \infty} \sum_{i=1}^{2} \sum_{q=\tau_{i}\left(n_{i}(j)\right)}^{n_{i}(j)-1} p_{i}(q) & =\liminf _{j \rightarrow \infty}\left[\sum_{q=12 j+4}^{12 j+4} p_{1}(q)+\sum_{q=12 j+2}^{12 j+3} p_{2}(q)\right] \\
& =\frac{17}{100}+2 \cdot \frac{89}{1,000}=0.348<\frac{1}{e}
\end{aligned}
$$

that is, condition (1.11) is not satisfied.

Example 3.2 Consider the advanced difference equation

$$
\nabla x(n)-p_{1}(n) x(n+1)-p_{2}(n) x(n+2)=0, \quad n \in \mathbb{N},
$$

where $p_{1}(n)$ and $p_{2}(n)$ are oscillating coefficients, as shown in Figure 2. 

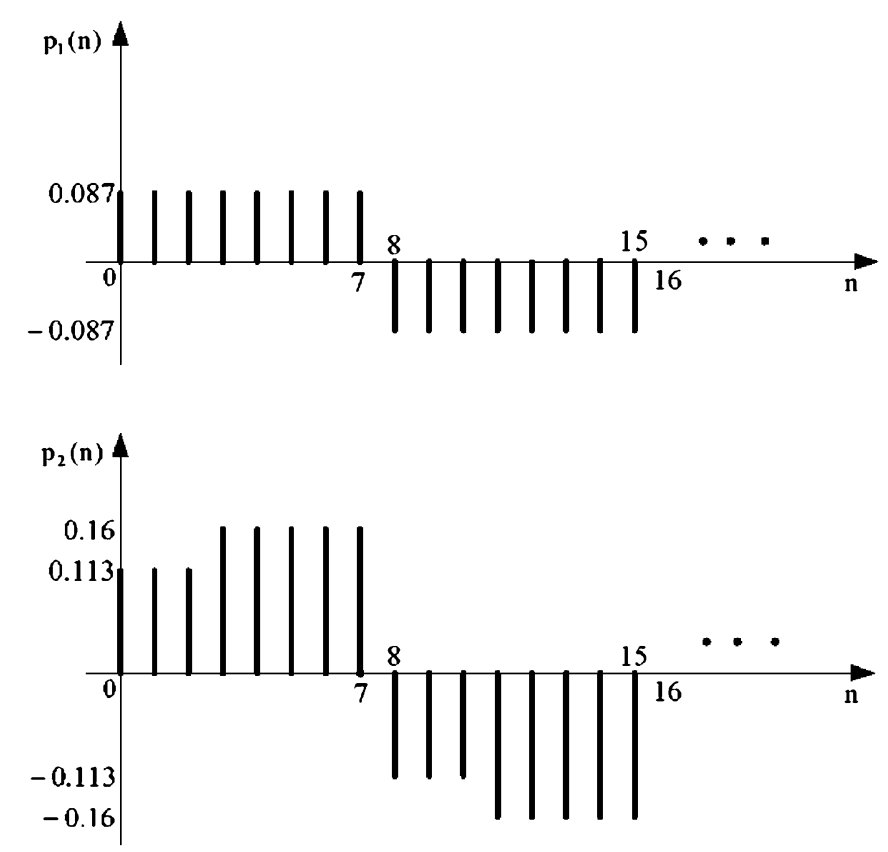

Figure 2 Coefficients of Example 3.2.

In view of (1.7), it is obvious that $\sigma(n)=n+1$. Observe that for

$$
n_{1}(j)=16 j+3, \quad j \in \mathbb{N},
$$

we have $p_{1}(n)>0$ for every $n \in A$, where

$$
A=\bigcup_{j \in \mathbb{N}}\left[n_{1}(j), \sigma\left(\sigma\left(n_{1}(j)\right)\right)\right] \cap \mathbb{N}=\bigcup_{j \in \mathbb{N}}[16 j+3,16 j+5] \cap \mathbb{N} .
$$

Also, for

$$
n_{2}(j)=16 j+1, \quad j \in \mathbb{N},
$$

we have $p_{2}(n)>0$ for every $n \in B$, where

$$
B=\bigcup_{j \in \mathbb{N}}\left[n_{2}(j), \sigma\left(\sigma\left(n_{2}(j)\right)\right)\right] \cap \mathbb{N}=\bigcup_{j \in \mathbb{N}}[16 j+1,16 j+3] \cap \mathbb{N} .
$$

Therefore

$$
p_{1}(n)>0 \quad \text { and } \quad p_{2}(n)>0 \quad \text { for all } n \in A \cap B=\{16 j+3\} \neq \emptyset \text {, }
$$

that is, (1.6) is satisfied. Moreover, the computation immediately implies that

$$
\frac{1}{2}\left(\sum_{i=1}^{2} \sqrt{p_{i} k_{i}}\right)^{2}=\frac{1}{2}\left(\sqrt{\frac{87}{1,000} \cdot 1}+\sqrt{\frac{16}{100} \cdot 2}\right)^{2} \simeq 0.370353228>\frac{1}{e},
$$


that is, condition (2.15) of Corollary 2.1 is satisfied and therefore all solutions of (3.2) oscillate. Observe, however, that

$$
\left[\prod_{i=1}^{2} p_{i}\right]^{1 / 2}\left(\sum_{i=1}^{2} k_{i}\right)=\sqrt{\frac{87}{1,000} \cdot \frac{16}{100}} \cdot(1+2) \simeq 0.353949148<\frac{1}{e}
$$

that is, condition (2.14) of Corollary 2.1 is not satisfied.

Observe that

$$
n(j)=\max \left\{n_{i}(j): 1 \leq i \leq 2\right\}=16 j+3, \quad j \in \mathbb{N} .
$$

Now

$$
\begin{aligned}
\alpha & =\liminf _{j \rightarrow \infty} \sum_{i=1}^{2} \sum_{q=n(j)+1}^{\sigma(n(j))} p_{i}(q) \\
& =\liminf _{j \rightarrow \infty}\left[\sum_{q=16 j+4}^{16 j+4} p_{1}(q)+\sum_{q=16 j+4}^{16 j+4} p_{2}(q)\right]=\frac{87}{1,000}+\frac{16}{100}=0.247 .
\end{aligned}
$$

Also

$$
\begin{aligned}
\limsup _{j \rightarrow \infty} \sum_{i=1}^{2} \sum_{q=n(j)}^{\sigma(n(j))} p_{i}(q) \\
\quad=\limsup _{j \rightarrow \infty}\left[\sum_{q=16 j+3}^{16 j+4} p_{1}(q)+\sum_{q=16 j+3}^{16 j+4} p_{2}(q)\right]=2 \cdot \frac{87}{1,000}+2 \cdot \frac{16}{100}=0.494 .
\end{aligned}
$$

Observe that

$$
\begin{aligned}
& 0.494<1 \\
& 0.494<1-\frac{\alpha^{2}}{4(1-\alpha)} \simeq 0.979744687 \\
& 0.494<1-\frac{1}{2}(1-\alpha-\sqrt{1-2 \alpha}) \simeq 0.979168384 .
\end{aligned}
$$

Therefore the conditions (1.8), (1.19), and (1.20) are not satisfied.

On the other hand, $p_{1}(n)>0$ for every $n \in A^{\prime}=A$ and $p_{2}(n)>0$ for every $n \in B^{\prime}$, where

$$
B^{\prime}=\bigcup_{j \in \mathbb{N}}\left[n_{2}(j), \sigma_{2}\left(\sigma_{2}\left(n_{2}(j)\right)\right)\right] \cap \mathbb{N}=\bigcup_{j \in \mathbb{N}}[16 j+1,16 j+5] \cap \mathbb{N}
$$

Therefore

$$
p_{1}(n)>0 \quad \text { and } \quad p_{2}(n)>0 \quad \text { for all } n \in A^{\prime} \cap B^{\prime}=\bigcup_{j \in \mathbb{N}}[16 j+3,16 j+5] \neq \emptyset,
$$


that is, condition (1.12) is satisfied. In view of this, we have

$$
\begin{aligned}
& \liminf _{j \rightarrow \infty} \sum_{i=1}^{m} \sum_{q=n_{i}(j)+1}^{\sigma_{i}\left(n_{i}(j)\right)} p_{i}(q) \\
& =\liminf _{j \rightarrow \infty}\left[\sum_{q=16 j+4}^{16 j+4} p_{1}(q)+\sum_{q=16 j+2}^{16 j+3} p_{2}(q)\right]=\frac{87}{1,000}+\frac{113}{1,000}+\frac{16}{100}=0.36<\frac{1}{e},
\end{aligned}
$$

that is, condition (1.14) is not satisfied.

\section{Competing interests}

The authors declare that they have no competing interests.

\section{Authors' contributions}

This paper is the result of the joint efforts of all the authors. All authors read and approved the final manuscript.

\section{Author details}

'Department of Electrical and Electronic Engineering Educators, School of Pedagogical and Technological Education (ASPETE), N. Heraklio, Athens, 14121, Greece. ${ }^{2}$ Faculty of Civil Engineering, University of Novi Sad, Kozaračka 2/A, Subotica, 24000, Serbia. ${ }^{3}$ Departamento de Ciências Exactas e Naturais, Av. Conde Castro Guimarães, Amadora, 2720-113, Portugal. ${ }^{4}$ Department of Mathematics, University of Ioannina, Ioannina, 451 10, Greece.

\section{Acknowledgements}

The research of Hajnalka Péics is supported by the Serbian Ministry of Science, Technology and Development for Scientific Research Grant no. III44006.

\section{Received: 29 September 2014 Accepted: 28 January 2015 Published online: 24 February 2015}

\section{References}

1. Berezansky, L, Chatzarakis, GE, Domoshnitsky, A, Stavroulakis, IP: Oscillations of difference equations with several oscillating coefficients. Abstr. Appl. Anal. 2014, Article ID 392097 (2014)

2. Bohner, M, Chatzarakis, GE, Stavroulakis, IP: Qualitative behavior of solutions of difference equations with several oscillating coefficients. Arab. J. Math. 3, 1-13 (2014)

3. Bohner, M, Chatzarakis, GE, Stavroulakis, IP: Oscillation in difference equations with deviating arguments and variable coefficients. Bull. Korean Math. Soc. 52(1), 159-172 (2015)

4. Chatzarakis, GE, Lafci, M, Stavroulakis, IP: Oscillation results for difference equations with several oscillating coefficients. Appl. Math. Comput. 251, 81-91 (2015)

5. Chatzarakis, GE, Péics, H, Stavroulakis, IP: Oscillation in difference equations with deviating arguments and variable coefficients. Abstr. Appl. Anal. 2014, Article ID 902616 (2014)

6. Fukagai, N, Kusano, T: Oscillation theory of first order functional-differential equations with deviating arguments. Ann. Mat. Pura Appl. 136, 95-117 (1984)

7. Grammatikopoulos, MK, Koplatadze, R, Stavroulakis, IP: On the oscillation of solutions of first-order differential equations with retarded arguments. Georgian Math. J. 10, 63-76 (2003)

8. Kulenovic, MR, Grammatikopoulos, MK: First order functional differential inequalities with oscillating coefficients. Nonlinear Anal. 8, 1043-1054 (1984)

9. Ladas, G, Sficas, G, Stavroulakis, IP: Functional-differential inequalities and equations with oscillating coefficients. In: Trends in Theory and Practice of Nonlinear Differential Equations. Lecture Notes in Pure and Appl. Math., vol. 90, pp. 277-284. Dekker, New York (1984)

10. Ladas, G, Stavroulakis, IP: Oscillations caused by several retarded and advanced arguments. J. Differ. Equ. 44, 134-152 (1982)

11. Li, X, Zhu, D, Wang, H: Oscillation for advanced differential equations with oscillating coefficients. Int. J. Math. Math. Sci. 33, 2109-2118 (2003)

12. Qian, C, Ladas, G, Yan, J: Oscillation of difference equations with oscillating coefficients. Rad. Mat. 8, 55-65 (1992)

13. Tang, $\mathrm{XH}$, Cheng, SS: An oscillation criterion for linear difference equations with oscillating coefficients. J. Comput. Appl. Math. 132, 319-329 (2001)

14. Xianhua, T: Oscillation of first order delay differential equations with oscillating coefficients. Appl. Math. J. Chin. Univ. Ser. B 15, 252-258 (2000)

15. Yan, W, Yan, J: Comparison and oscillation results for delay difference equations with oscillating coefficients. Int. J. Math. Math. Sci. 19, 171-176 (1996)

16. Yu, JS, Tang, XH: Sufficient conditions for the oscillation of linear delay difference equations with oscillating coefficients. J. Math. Anal. Appl. 250, 735-742 (2000)

17. Yu, JS, Zhang, BG, Qian, XZ: Oscillations of delay difference equations with oscillating coefficients. J. Math. Anal. Appl. 177, 432-444 (1993) 